RHIC-PH-4

RHIC AND QUARK MATTER: A PROPOSED HEAVY ION COLLIDER AT

BROOKHAVEN NATIONAL LABORATORY

T. Ludlam

Brookhaven Nationa1 Laboratory

June 1984 
BNL -35237

\title{
RHIC AND QUARR MATTER: A PROPOSED HEAVY ION COLLIDER AT BROOKHAVEN RATIONAL LABORATORY
}

\author{
Presented by \\ Thomas W. Ludlam \\ Brookhaven National Laboratory \\ Upton, NY 11973, USA \\ QUARR MATTER ' 84 \\ Fourth International Conference on \\ U1trarelativistic Nucleus-Nucleus Collisions \\ Helsinki, Finland \\ June $17-21,1984$
}

The submitted manuscript has been authored under contract DE-AC02-76CH00016 with the U.S. Department of Energy. Accordingly, the U.S. Government retains a nonexclusive, royalty-free license to publish or reproduce the published form of this contribution, or allow others to do so, for U.S. Government purposes. 
RHIC AND QUARK MATTER: A PROPOSED HEAVY ION COLLIDER AT BROOKHAVEN NATIONAL LABORATORY

\author{
T. Ludlam \\ Brookhaven National Laboratory \\ Upton, NY 11973/USA
}

I. Introduction

In August, 1983 a Working Group of nuclear and particle physicists was convened at Brookhaven to consider the basic parameters for a high energy heavy ion collider facility. The meeting was in response to a unique set of circumstances arising from the termination of the CBA project and the result of the NSAC long range planning study ${ }^{l}$ in which an ultra-relativistic heavy ion collider was identified as the important new scientific opportunity for U.S. nuclear physics.

For some time there had been strong interest at Brookhaven in exploiting the existence of the Colliding Beam Accelerator, CBA (nee ISABELLE), for the generation of heavy ion collisions at very high energies. 2 Now the opportunity arose to consider the design of a dedicated heavy ion collider which could take advantage of the work already invested in the CBA project.

In its report ${ }^{3}$ the working Group recommended a top energy of at least $50+50 \mathrm{GeV} / \mathrm{amu}$, emphasizing the need to cover a continuous range of energies extending as low $5+5 \mathrm{GeV} / a \mathrm{mu}$, with ion masses ranging from protons up to $\dot{A}>200$, and luminosity $L>10^{25} \mathrm{~cm}^{-2} \mathrm{sec}^{-1}$ initially, ultimately reaching much higher values.

These parameters were the subject of extensive scrutiny at the Quark Matter ' 83 Conference ${ }^{4}$ one month later at which several of the world's laboratories presented possibilities for very high energy nuclear beam facilities. After several days of such discussions a round table panel at the Conference ${ }^{5}$ strongly endorsed the recommendations of the August Working Group, with an emphasis on making the top energy as high as possible without sacrificing the capability for exploring the low energy range as well.

In the months since the Quark Matter 183 Conference an intensive design effort has been carried out at Brookhaven for a dedicated Relativistic Heavy Ion Collider (RHIC) using the existing site, 
tunnel, cryogenic refrigerator and experimental areas of the CBA project. During the course of this study a number of heretofore unappreciated features of accelerator physics came to light regarding the special case of storing intense beams of highly charged ions. 6 Most important of these is the phenomenon of intrabeam scattering ${ }^{7}$ which imposes strong limitations on the performance of a heavy ion collider. Nonetheless, the requirements outlined above can be met.

The Brookhaven RHIC design was completed and submitted as a preliminary proposal ${ }^{8}$ to the U.S. 'Department of Energy during June, 1984. It calls for a top beam energy of $100 \mathrm{GeV} / \mathrm{amu}$ for $A=200$, and the acceleration of ion masses spanning the full periodic table. The proposed accelerator complex utilizes the existing Tandem Van de Graaff and $A G S$ as source and injector, and will provide center-of-mass collision energies from $1.5+1.5 \mathrm{GeV} / \mathrm{amu}$ to 100 $+100 \mathrm{GeV} / \mathrm{amu}$.

This range is covered, with no gaps, using the AGs beams for fixed target experiments $(\sqrt{\mathrm{s}} / \mathrm{A}<5 \mathrm{GeV} / \mathrm{amu})$; one collider beam striking an internal gas jet or thin wire target ( $5 \mathrm{GeV} / a m u<\sqrt{ } / \mathrm{A}<14$ $\mathrm{GeV} / \mathrm{amu}$ ), and the colliding beams of the Collider itself (14 GeV/amu - $<\sqrt{\mathrm{s}} / \mathrm{A}<200 \mathrm{GeV} / \mathrm{amu})$. The complete facility will thus provide heavy ion collisions over a spectrum of energies which begins just above the present Bevalac, extends through the range of "maximal stopping" 9 "were high energy density is achieved at high baryon number, and on up to the extremely high energies now being glimpsed in rare cosmic ray events.

In the high energy regime achieved with the collider we expect a fully developed central rapidity region: an environment as close as we can achieve in the laboratory to that of the very early universe and the baryon-free conditions assumed in gauge theory calculations of finite temperature $Q C D$ and in hydrodynamic theories of the evolution of a quark-gluon plasma. At these energies we have access to a regime in which we know the individual nucleon bags can be penetrated, dislodging the constituent quarks and gluons. Here a potentially powerful probe becomes accessible in the form of jets observed after propagating through the new matter created in the collision. 10

Fig. I shows the range of energies and kinematic terrain covered by the AGS and RHIC complex. In the following sections we discuss the detailed design parameters and performance specifications. 


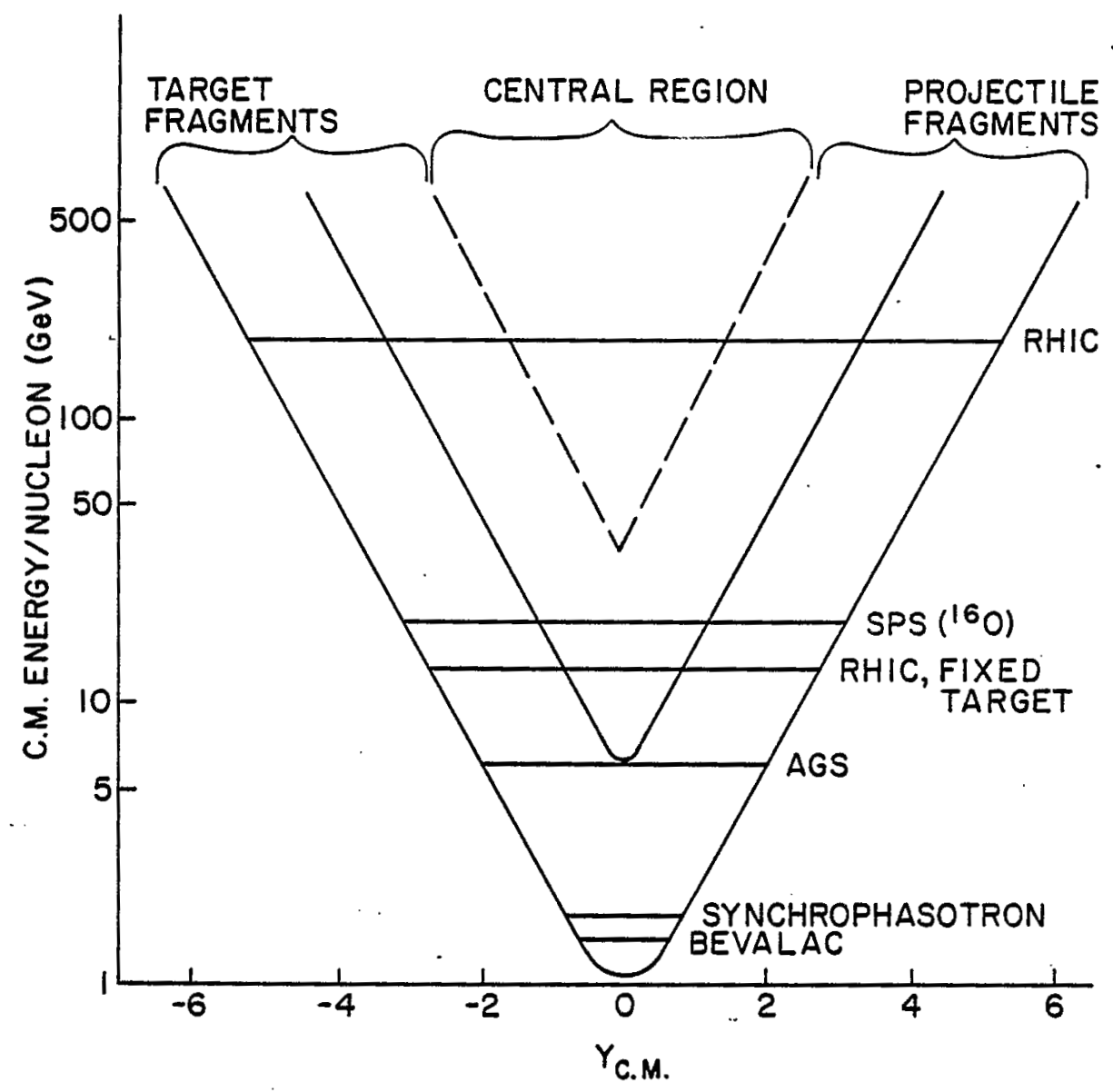

Figure 1.

The kinematic landscape available to the RHIC facility, and some existing machines. The outer "vee" is the phase space Iimit--the rapidity of the incident nucleons. The inner, solid vee delineates fragmentation regions of width $\Delta y=2$, as observed in protonproton collisions. The dashed lines indicate the wider $(\Delta y=4)$ fragmentation regions--and hence narrower central region--expected at a given energy for nucleusnucleus collisions with $A \approx 200$. The horizontal lines indicate energies reached with the components of the AGS/RHIC complex, as well as in the facilities which presently exist (LBL Bevalac, Dubna Synchrophasotron) and the planned oxygen, beams in the CERN SPS. 
II. The RHIC Design

II. I Source and Injection

Figure 2 is a site plan of the Brookhaven accelerator complex, showing the major components of the RHIC facility. The existing Tándem Van de Graaff accelerators will serve as source and initial ion accelerator. The ions will then traverse a long $(\approx 2000$ ft) transfer line to a small Booster synchrotron, from which fully stripped ions at an energy of $\approx 320 \mathrm{MeV} / a m u$ are injected to the AGS. Each particle bunch transferred from the Booster to the AGS is accelerated to the top AGS energy $(28 \mathrm{GeV}$ for protons; $11 \mathrm{GeV} / a m u$ for gold) and then transferred to the collider by a magnet system installed in the existing injection line tunnels.

Construction of the transfer line from the Tandem accelerators to the AGS has now begun, as part of a program to accelerate ion beams in the AGS for fixed target experiments beginning in 1986.11 These experiments will initally have thë Tandem alone as ion source, providing fully stripped ions for injection directly into the AGs up to sulfur $(A=32)$. Higher masses require the Booster. Brookhaven has requested funds for the Booster as part of a general improvement of the AGS performance for high energy physics experiments. If it is funded construction is expected to begin in 1986, making it operational in 1988 .

The Tandem Van de Graaf provides beams of very small transverse emittance (< $1 \pi \mathrm{mm} \mathrm{mrad}$ at exit), with an energy stability of one part in $10^{4}$, and so is well suited for injecting heavy ions into a synchrotron. Using a new type of sputter ion source ${ }^{8}$ the machine can be operated in a pulsed mode with currents up to $200 \mu \mathrm{A}$. The ions pass through two stripping foils before arriving at the booster, where Gold is in charge state +33 .

Table 1 shows the characteristics of the beam injected into the Booster for a representative sample of ion species. Here $\varepsilon_{\mathbb{N}}$ is the normalized emittance and $N_{B}$ is the total number of particles in one Booster fill, which will be transferred to the AGS as one bunch. The maximum number of particles which can be injected to the Booster (the space charge limit) is given as NSC in Table 1. It will be seen that the beam intensity is limited by the Tandem currents for the Iighter ions, up to Copper, while for the heavier ions the maximum intensity is determined by space charge limits. After extraction from the booster and on their way to the AGS the ions pass through one more stripping target. The ions injected into the AGS are then fully stripped. 


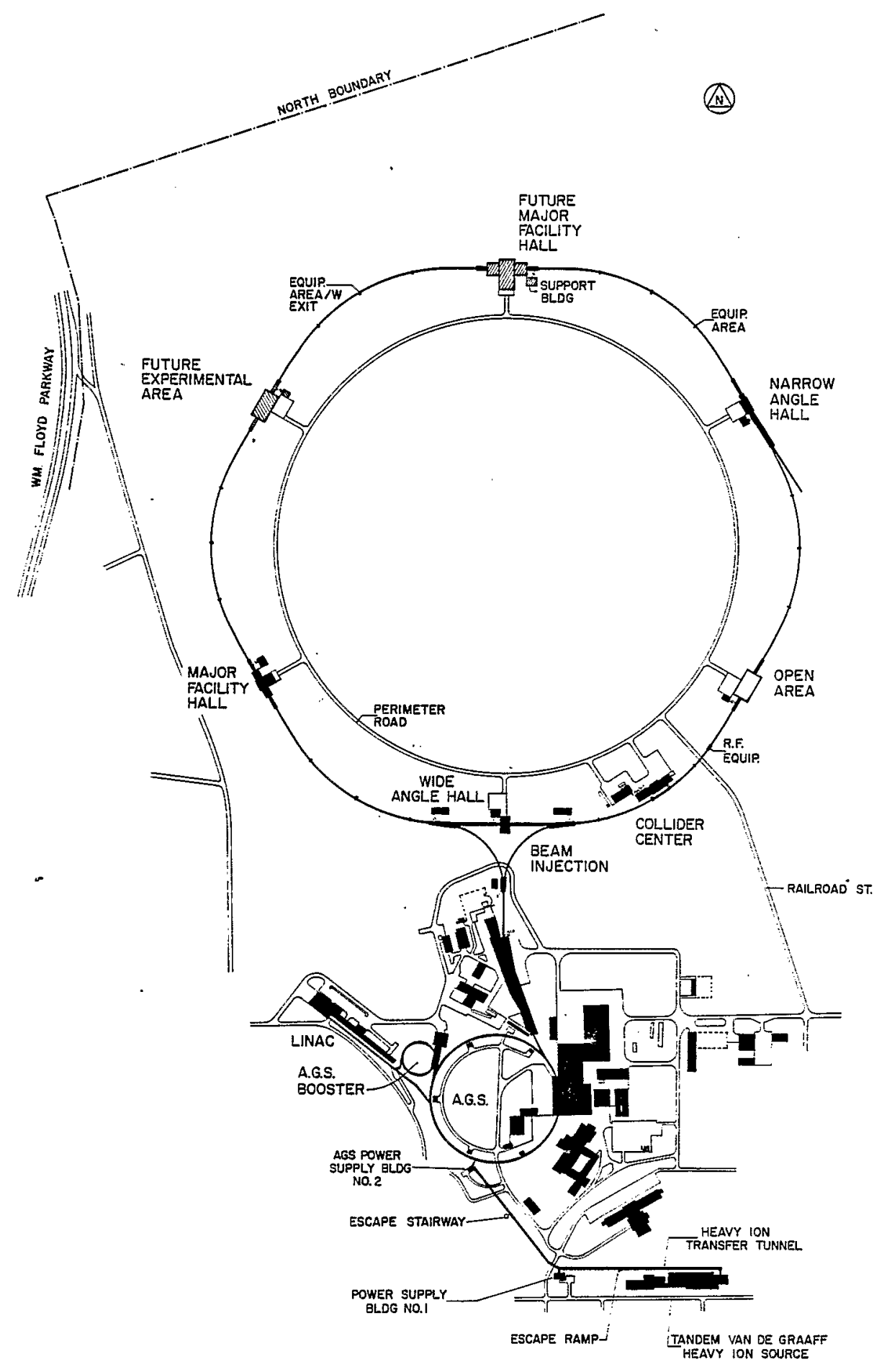

SITE MAP

Figure 2 .

Site Layout of the $\bar{R} H I C$ Project. 
Table 1

Beam Intensity, Emittance of Injection into the Booster

\begin{tabular}{lcccc}
\hline Element & $\begin{array}{c}\varepsilon_{\mathrm{N}} \\
\pi \cdot \mathrm{mm} \cdot \mathrm{mrad}\end{array}$ & $\begin{array}{c}\mathrm{T}_{\mathrm{rev}} \\
\mu \mathrm{sec}\end{array}$ & $\mathrm{N}_{\mathrm{B}} \times 10^{9^{*}}$ & $\mathrm{~N}_{\mathrm{SC}} \times 10^{9^{* *}}$ \\
\hline Deuterium & 8.8 & 3.81 & 100.0 & 438.0 \\
Carbon & 6.3 & 5.33 & 22.0 & 37.0 \\
Sulphur & 6.0 & 6.72 & 6.7 & 11.0 \\
Copper & 3.9 & 8.60 & 4.7 & 5.5 \\
Iodine & 3.0 & 11.31 & 3.4 & 3.2 \\
Gold & 2.3 & 14.53 & 3.6 & 2.2 \\
\hline
\end{tabular}

*With 8-turn injection

$* *_{\text {At }} \quad \Delta v=.1$

Since the ions are completely stripped upon injection to the AGS, there is no requirement for improvement of either the vacuum or the rf system of the present accelerator. Ions will be transferred one bunch at a time from the Booster to the AGS, with each bunch then accelerated to the top energy of the AGS and sent to the collider. The cycle time for this process is $0.8 \mathrm{~Hz}$.

\section{II.2 The Collider}

The layout of the collider is illustrated in Fig. 3 . it is composed of two identical concentric rings in a common horizontal plane. There are six crossing points. 


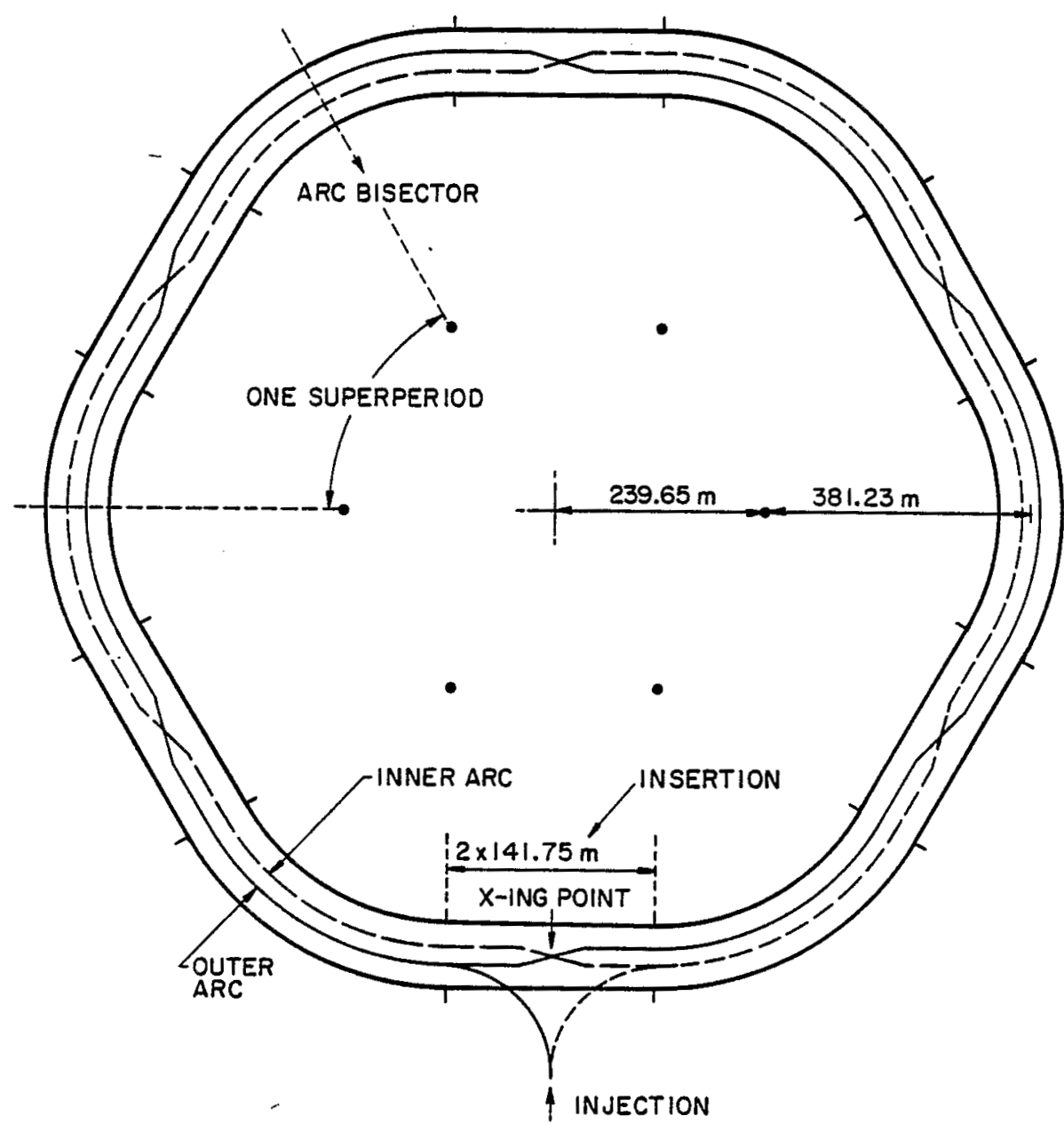

Geometry of the Collider Beams.

Each ion Bunch accelerated in the AGS is extracted and transferred to one of the two Collider rings. The bunch is then captured by a stationary rf bucket. The two rings are filled in box-car fashion, with a total of 57 bunches per ring. An equivalent number of AGS pulses is required which gives a filling time a little more than one minute per ring. The bunch separation is 67 meters, or about 200 nanoseconds. The major parameters of the collider rings are given in Table 2.. Some properties of the beams, at injection, are given in Table 3 . 
Table 2

General Parameters for the Collider

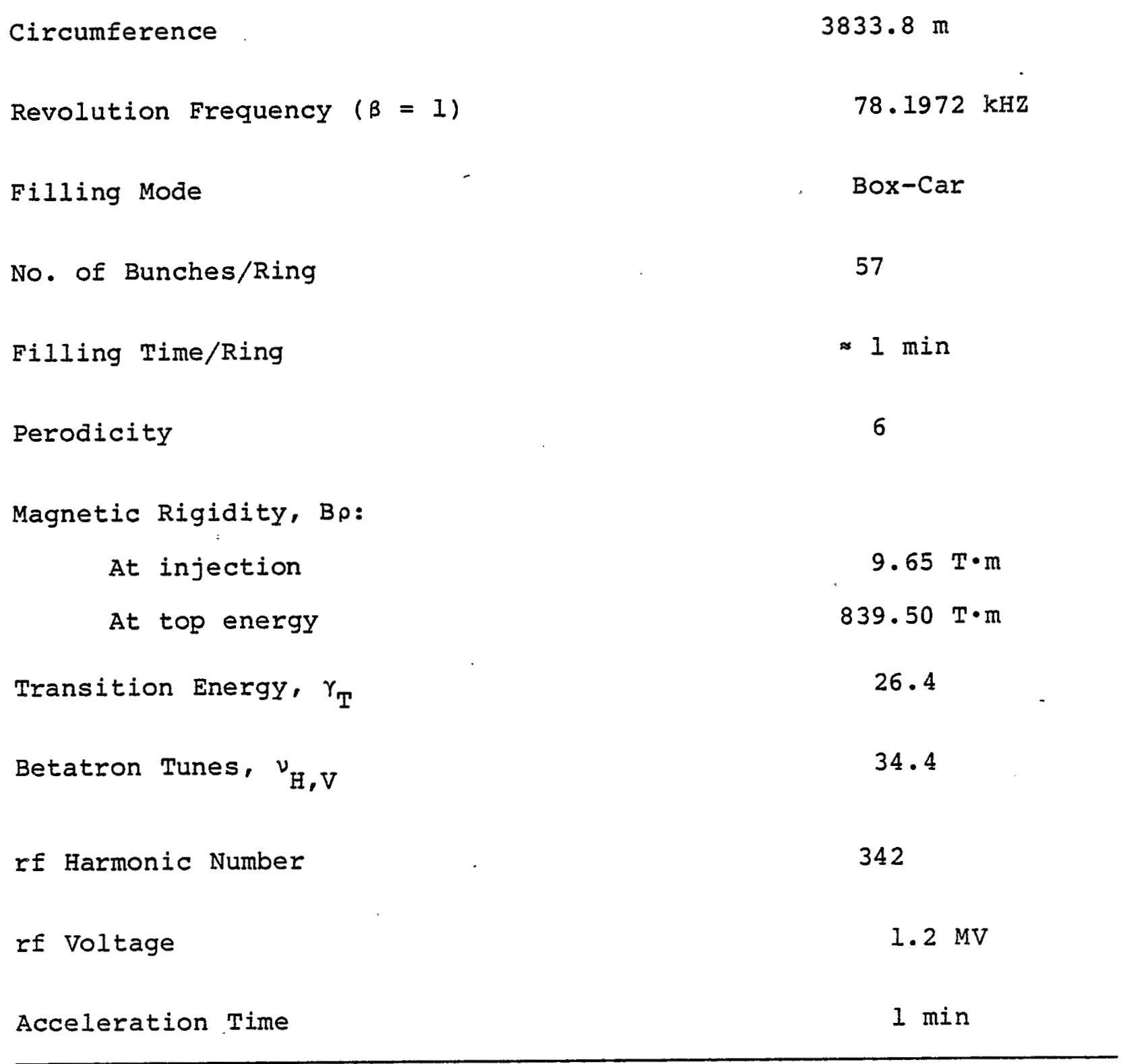


Table 3

General Beam Parameters for the Collider

\begin{tabular}{|c|c|c|c|c|c|c|c|}
\hline Element & Proton & $\begin{array}{l}\text { Deuter- } \\
\text { ium }\end{array}$ & Carbon & Sulphur & Copper & Iodine & Gold \\
\hline \multicolumn{8}{|l|}{$\begin{array}{l}\text { Injection: } \\
\text { Kinetic }\end{array}$} \\
\hline Energy, Gev/A & 28.0 & 13.6 & 13.6 & 13.6 & 12.4 & 11.2 & 10.7 \\
\hline $\begin{array}{l}\text { Bunch Length } \\
\text { nsec }\end{array}$ & \pm 8.6 & \pm 8.6 & \pm 8.6 & \pm 8.6 & \pm 8.6 & \pm 8.6 & \pm 8.6 \\
\hline $\begin{array}{l}\text { No. ions } / \\
\text { Bunch, } x 10^{9}\end{array}$ & 1000 & 100. & 22 & 6.4 & 4.5 & 2.6 & 1.1 \\
\hline \multicolumn{8}{|l|}{ Top Energy: } \\
\hline \multicolumn{8}{|l|}{$\begin{array}{l}\text { Luminosity: } \\
\left(\mathrm{cm}^{-2} \sec ^{-1}\right)\end{array}$} \\
\hline $\begin{array}{l}\alpha=0 \text { mrad: } \\
\alpha=2 \text { mrad: }\end{array}$ & $1.2 \times 10^{31}$ & $1.2 \times 10^{31}$ & $\begin{array}{l}6 \times 10^{29} \\
.23 \times \alpha\end{array}$ & $\begin{array}{l}5 \times 10^{28} \\
0 \text { Values }\end{array}$ & $2 \times 10^{28}$ & $7 \times 10^{27}$ & $1.2 \times 10^{27}$ \\
\hline
\end{tabular}

*Initial Luminosity at injection. $\alpha=$ beam crossing angle.

The insertion regions are designed to provide some measure of flexibility in the focussing parameters at the crossing points, so that each insertion may be optimized for a particular experiment or machine function. The half cell insertion layout is shown in Fig. 4. The dipoles $B C I$ and $B C 2$ are used to define the crossing angle $\alpha$; $\mathrm{BCl}$ is common magnet for both beams. A free space of 11 meters on either side of the crossing point is available for detector equipment. 


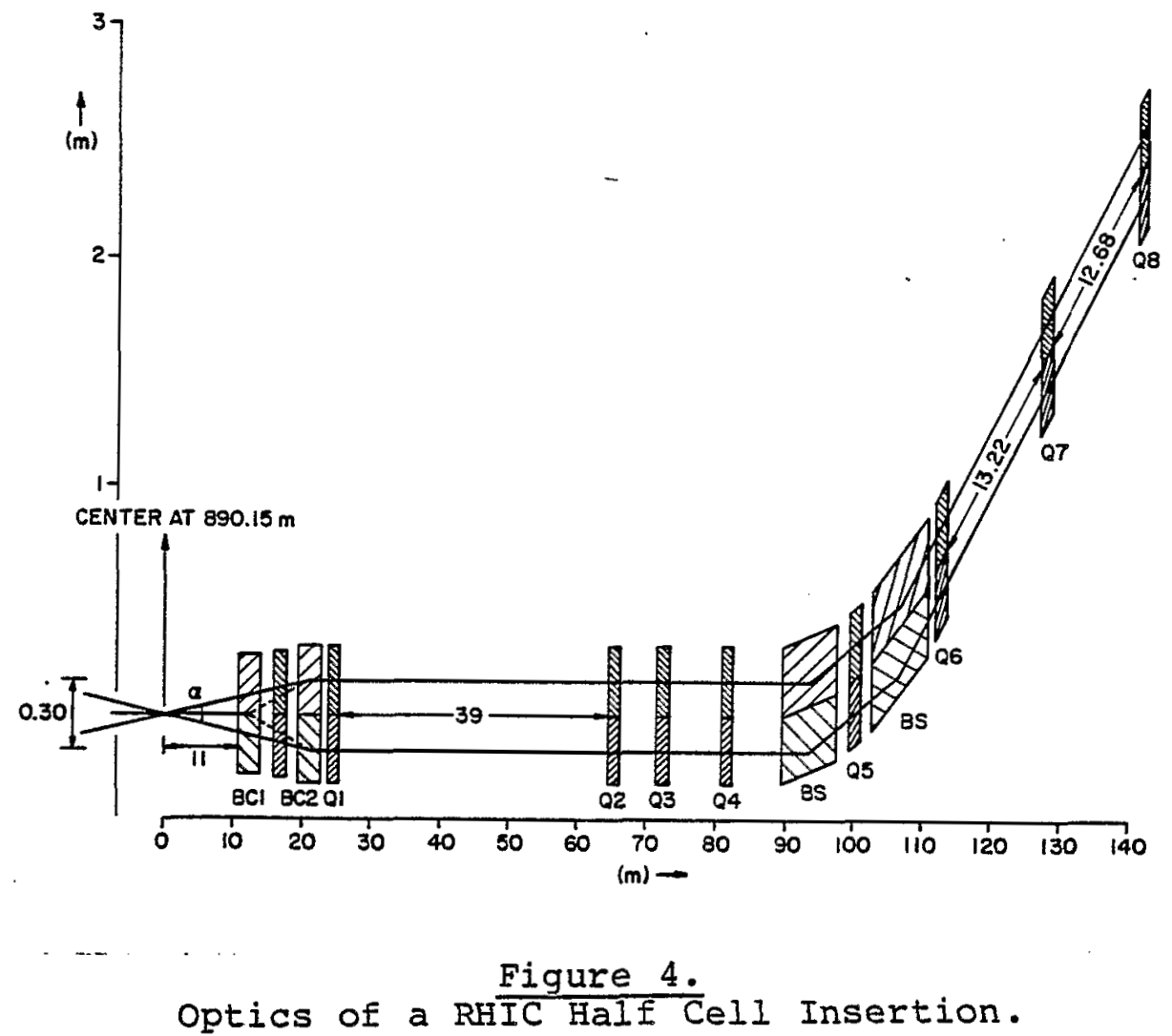

Operation with beams in head-on collisions $(\alpha=0)$ gives the maximum luminosity, and also the maximum length of the luminous diamond. For $\mathrm{Au}$ beams at top energy the rms diamond length is $\approx 20$ $\mathrm{cm}$ at injection, and grows by nearly a factor of 3 , due to intrabeam scattering, after 10 hours of operation. With a crossing angle $\alpha=$ $2 \mathrm{mr}$ the diamond size can be reduced, for those experiments which require it, by a factor of $\approx 4$, with corresponding reduction in luminosity (see Table 3 ).

The insertion scheme allows for operation with unequal ion species in the two beams. For example, operation with protons in one ring and gold ions in the other can be carried out with luminosity $1.2 \times 10^{29} \mathrm{~cm}^{-2} \mathrm{sec}^{-1}$.

The most severe limitation for maintaining intense beams of heavy ions over long periods of time is the phenomenon of intrabeam scattering. ${ }^{6-8}$ This effect, by which particles in the same bunch exchange longitudinal and transverse momenta through Coulomb scattering, depends strongly on the charge-to-mass ratio, being proportional to $\mathrm{Z}^{4} / \mathrm{A}^{2}$. The phenomenon has been observed in electron and proton storage rings, and is now understood to be the dominant cause of beam diffusion for heavy ion colliders. (By way of comparison, space charge effects such as the beam-beam tune shift and microwave instability go as $\mathrm{z}^{2} / \mathrm{A}$.) 
Table 4

Experimental Hall Dimensions (Meters)

\begin{tabular}{|c|c|c|c|c|}
\hline & Length & Width & $\begin{array}{l}\text { Beam } \\
\text { Height }\end{array}$ & $\begin{array}{l}\text { Hook Height \& } \\
\text { Capacity (tons) }\end{array}$ \\
\hline \multicolumn{5}{|l|}{2 o'clock } \\
\hline Central Hall & 28 & 12.0 & 1.7 & $6.1 / 20$ \\
\hline Foward Hall & $68^{\circ}$ & 7.9 & 1.7 & $5.3 *$ \\
\hline "Stub" & 91 & 2.4 & 1.0 & $2.0 *$ \\
\hline \multicolumn{5}{|l|}{$\underline{4} 0^{\prime}$ clock } \\
\hline Open Area Pad & 57 & 37.0 & 2.2 & --- \\
\hline \multicolumn{5}{|l|}{6 o'clock } \\
\hline Wide Angle Hall & 16 & 32.0 & 4.3 & $10 / 2 \times 20$ \\
\hline \multicolumn{5}{|l|}{$80^{\prime} \mathrm{clock}$} \\
\hline Major-Facility Hall & 19. & 15.0 & 5.2 & $11 / 40$ \\
\hline 2 Forward Halls & 16 & 9.0 & 3.3 & $6.6^{*}$ \\
\hline Assembly Building & 19 & 19.0 & 5.2 & $11 / 40+14 / 7.5$ \\
\hline
\end{tabular}

*No Crane: Ceiling height given. 
The availability of completed construction work and other assets from the ISABELLE/CBA project represents an unprecedented opportunity to build, at minimal cost, a dedicated heavy ion facility with beam energies up to $100 \mathrm{GeV} / \mathrm{amu}$. These assets include more than just the ring tunnel. They include also: a complete injection system (the Tandem-Booster-AGS Complex) which will exist independently of the collider; the injection tunnels from the AGS; three completed experimental areas (three more undeveloped experimental areas also exist); a central control building. The cryogenic refrigerator system designed to cool the CBA magnets is complete. In addition, Brookhaven has an experienced superconducting magnet group and very large facilities for constructing, assembling and testing superconducting magnets. The four intersection regions which have already in place the structures necessary for an operating experimental area are indicated in Fig. 2. These areas are at the 2, 4, 6 and 8 o'clock locations. The area at 4 o'clock is an "open area," with a completed concrete pad and auxiliary building. The remaining areas have fully enclosed experimental halls with overhead cranes. The sizes of these experimental areas are given in Table 4. The 10 and 12 o'clock experimental areas were left undeveloped when the CBA project was terminated, and will not be available for first-round RHIC experiments.

The cost estimate obtained for the RHIC proposal assumes the completion of the Tandem-AGS transfer line, which is now under construction, and the Synchrotron Booster, which is planned to start in FY 1986. The total cost estimate for the Collider, including contingency, is $\$ 134.4 \mathrm{M}$ in 1984 dollars. This assumes a conservative schedule of 4 years for magnet production (of which the first year goes to R\&D and fabrication of tooling) and 5 years for overall project completion. With this schedule, beams could be available for the first experiments during the latter half of 1990.

\section{Acknowledgement}

As I have indicated, the development of the RHIC proposal has benefitted from the advise and consultation of a very large number of interested physicists representing laboratories throughout the world. the technical design effort has involved many members of the High Energy Facilities division at BNL, under the direction of 
M.Q. Barton and H. Hahn. This group is pleased to acknowledge the significant contributions of A. Ruggiero (Fermilab) and G. Young (ORNL).

This work was supported by the U.S. Department of Energy under contract DE-AC02-76-CH00016.

References

1. "A Long Range Plan for Nuclear Science," A Report by the DOE/NSF Nuclear Science Advisory Committee, December 1983.

2. H.A. Gordon (Ed.), Proceedings of the 1981 ISABELLE Summer Workshop, BNL 51443 (1981); M.Q. Barton, "The Acceleration of Heavy Ions to Very High Energies," Proc. Bielefeld Workshop on Quark Matter Formation and Heavy Ion Collisions (M. Jacob and H. Satz, eds), Word Scientific, Singapore (1982); T.W. Ludlam, "Heavy Ion Physics at CBA," BNL 32716 (1983).

3. "Report of the Task Force for Heavy Ion Physics," Ref. 4, below p. 657 .

4. T.W. Ludlam and H.E. Wegner (Eds.), Proceedings of the Third International Conference on Ultra-Relativistic Nucleus-Nucleus Collisions: Quark Matter 183, Nucl. Phys. A418 (1984).

5. "Round Table Discussion of Prospects for Future Experiments." D.A. Bromley, Chairman), Ref. 4, p. 375.

6. G. Young, "Heavy Ion Colliders," Proc. Conference on the Intersections between Particle and Nuclear Physics," May, 1984 Steamboat Springs, Colorado (R. Mischke, Ed.), (To be Published).

7. A. Ruggiero, "Intrabeam Scattering and Heavy Ion Colliders," G. Young, "Heavy Ion Colliders," Proc. Conference on the Intersections between Particle and Nuclear Physics," May, 1984 , Steamboat Springs, Colorado (R. Mischke, Ed.) (To be Published).

8. "Proposal for a Relativistic Heavy Ion Collider at Brookhaven National Laboratory," BNL 51801 (June, 1984).

9. W. Busza, These Proceedings; see also W. Busza, Ref. 4, p. 635 .

10. T. Ludlam, I. Madansky, F. Paige, "High Energy Probes of the Quark-Gluon Plasma," Proc. of the LBI Workshop on Detectors for Relativistic Nuclear Collisions, March, 1984 (L. Schroeder, Ed.), To be published.

11. K. Koley, These Proceedings.

12. I. Schroeder, Ed., Proceedings of the LBL Workshop on Detectors for Relativistic Nuclear Collisions, March, 1984 (To be Published).

13. O. Miyamura, These Proceedings; See also T. Burnett, et al., Phys. Rev. Lett. 50, 2062 (1983). 


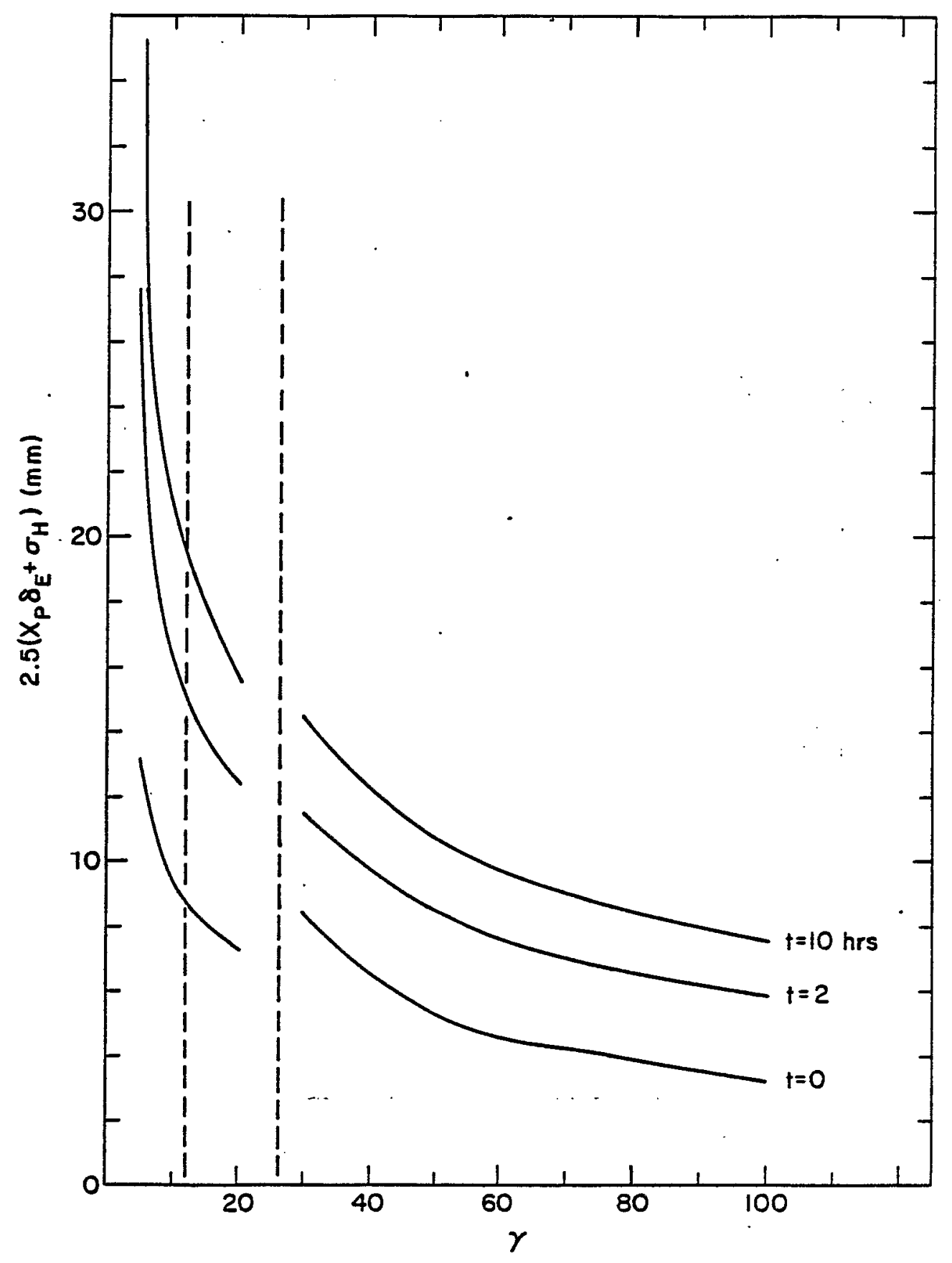

Figure 5 .

The half-width for $95 \%$ beam containment for gold ions, as a function of the beam energy, showing the growth with time due to intrabeam scattering. 
The effect of intrabeam scattering on beams of gold ions in the RHIC lattice is illustrated in Fig. 5. This shows the overall beam dimension as a function of the energy at injection $(t=0)$, and at the end of two-hour and ten-hour storage periods. It will be seen that for energies below transition $\left(\gamma_{\mathrm{T}}=26.4\right)$ the beams become quickly very large, whereas the beam dimension varies by no more than a factor of two for energies above $\gamma_{T}$. It is the size of the beam envelope, as shown in Fig. 5, which must determine the aperture of the collider magnets. The "good field" region of either quadrupoles or dipoles should be large enough to contain the beam envelope for the required range of energies and storage times. The choice of magnet aperture has a direct influence on the overall cost of the machine as well. For the RHIC design it was decided to set the aperture to adequately contain the beam for energies above $\gamma=$ 30, after 10 hours of storage. Useful beams are still available down to $\gamma \simeq 7$, but with shorter beam lifetimes. At $\gamma=7$ the total collision energy is $7+7 \mathrm{GeV} / \mathrm{amu}$, equal to the cm energy of a single $\gamma=100$ beam striking a fixed target. Thus the energy range below that which is accessible in the collider mode can be covered by operating a single ring with one or more internal fixed targets. Suitable targets, with thickness $\approx 10^{-9} \mathrm{gm} \mathrm{cm}^{-2}$, can be achieved with gas jets or thin wires. In this way the full range, from the lowest AGS energy to the top collider energy can be studied, with no inaccessible gaps. The luminosity which is achieved for the various modes of operation over this energy interval is shown in Fig. 6 . Each collider ring has approximately 200 dipole and 250 quadrupole magnets. These will be superconducting magnets of a design optimized for the heavy ion application, and so will be quite different from the magnets designed for CBA. Because of the lower beam intensity and energy, and a machine design which assumes a short ce11, "strong" focussing lattice, the RHIC'magnets will be longer $(\approx 10 \mathrm{~m})$, of smaller aperture, and have significantly lower fields (3.5 T at top energy) than was the case for the CBA design. All of these differences contribute to a lower cost for the RHIC magnet system. Candidate solutions now undergoing detailed study call for adjacent magnets in the two rings to share the same vacuum vessel, but without magnetic coupling. These "dual" magnets utilize a single layer dipole design based on experience with the CBA magnets, as well as dipoles under consideration by the Brown-Boveri Corporation for the HERA project at DESY, and dipoles under study for the SSC. 


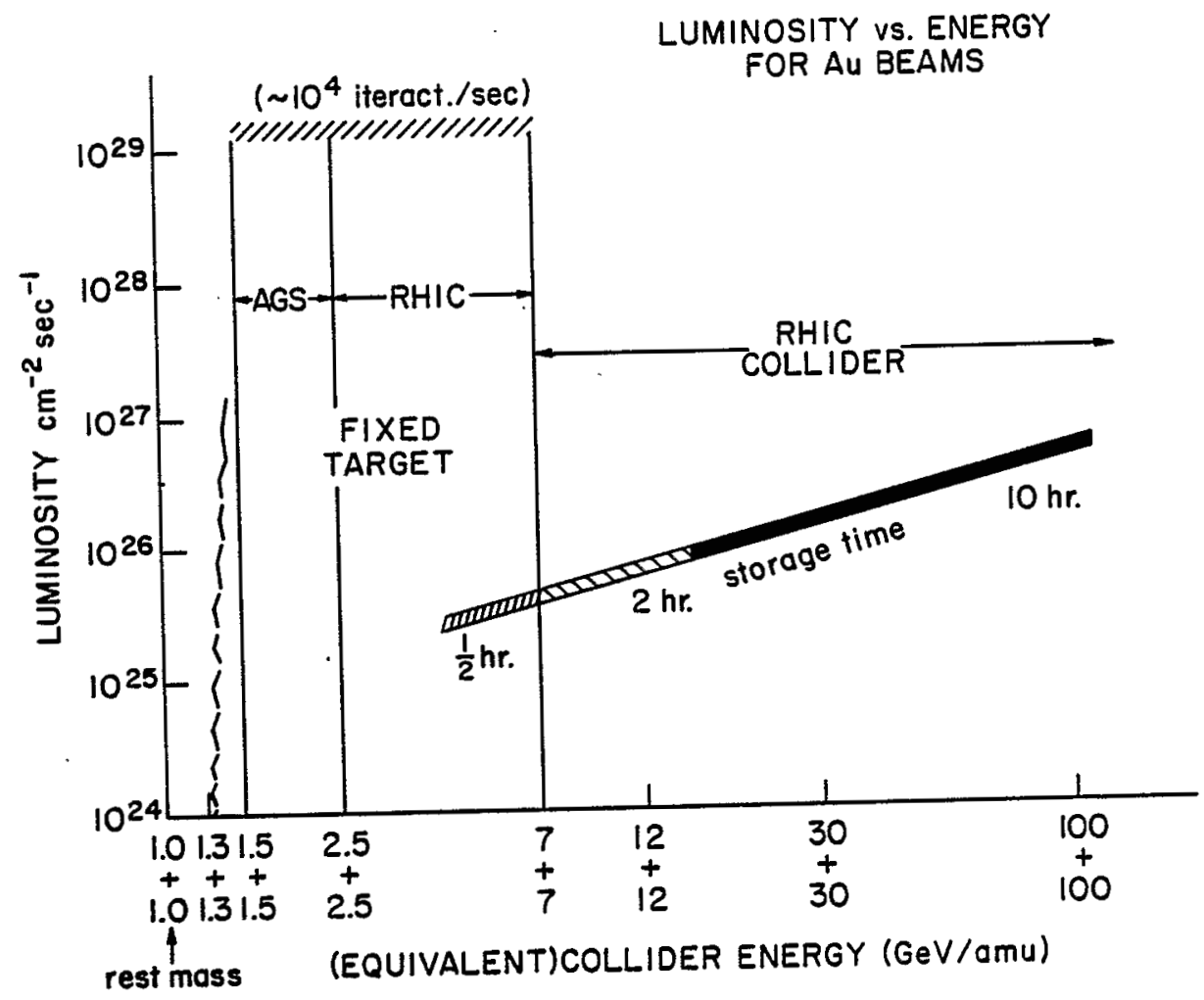

Figure 6.

The average Iuminosity for ${ }^{197} \mathrm{Au}$ beams over the full range of energy accessible with AGS and RHIC.

\section{Experiments and Detectors}

An experimental program to explore all of the facets of this new physics implies a measurement capability similar to that which we see in spectrometers for high energy elementary particle experiments. The design of instrumentation for experiments with ultrarelativistic nuclear beams has recently been the subject of extensive workshop activity, $2,3,12$ and these discussions have included large, general purpose facilities as well as smaller, more specialized detector systems. Figure 7 a shows very roughly the geometry and kinematics of a large solid angle detector, designed for use with colliding beams and having tracking and calorimetery in the central rapidity region and at forward angles. A real detector system might have more limited solid angle coverage. Note that the number of particles in each of the indicated rapidity intervals is expected to be many hundreds. The RHIC insertion design and the experimental halls (three of which are already completed) allow for detector systems of this scale. 


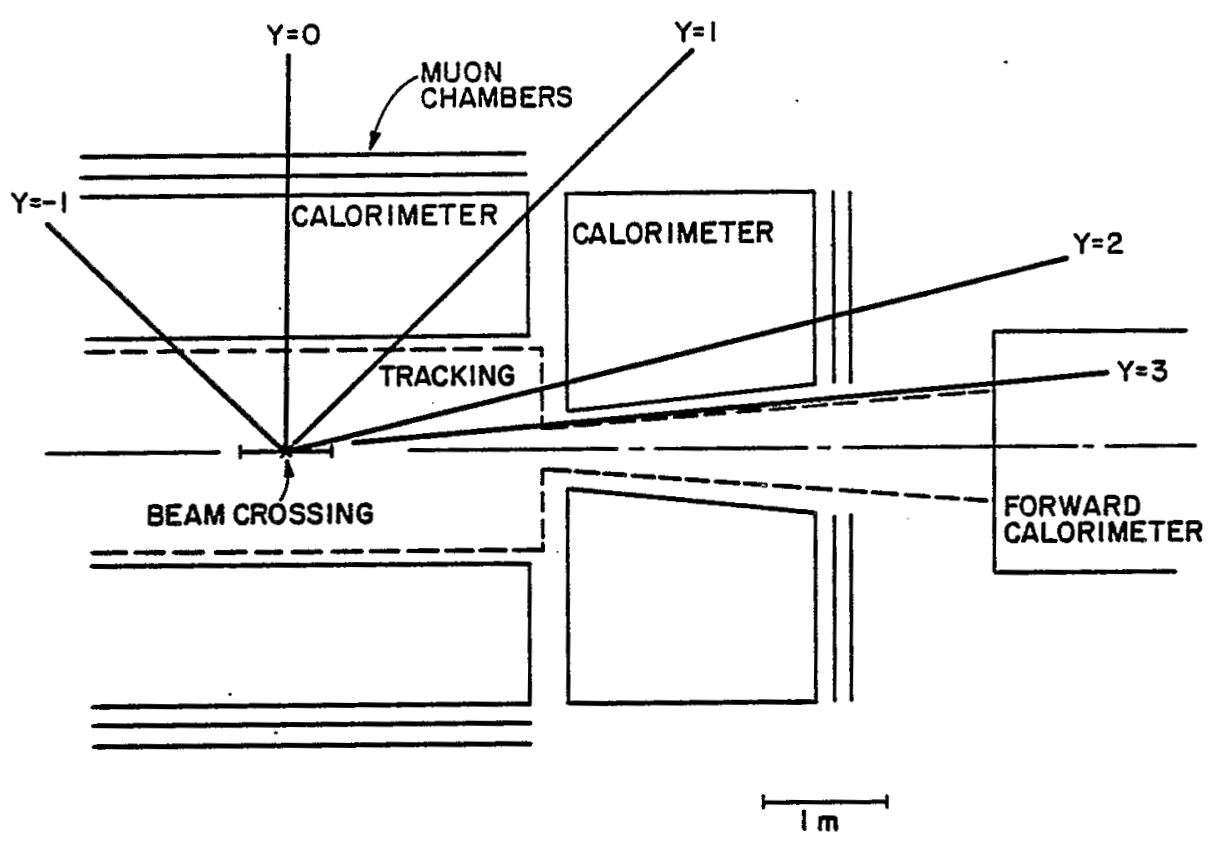

Figure 7.

(a) Schematic layout showing the geometry and kinematics of a detecting system for colling beam experiments. The angular intervals corresponding to various rapidity ( $y$ ) values are indicated. A full solid-angle detector would have the same coverage on either side of $y=0$; this illustration shows coverage of the central region and one of the fragmentation regions.

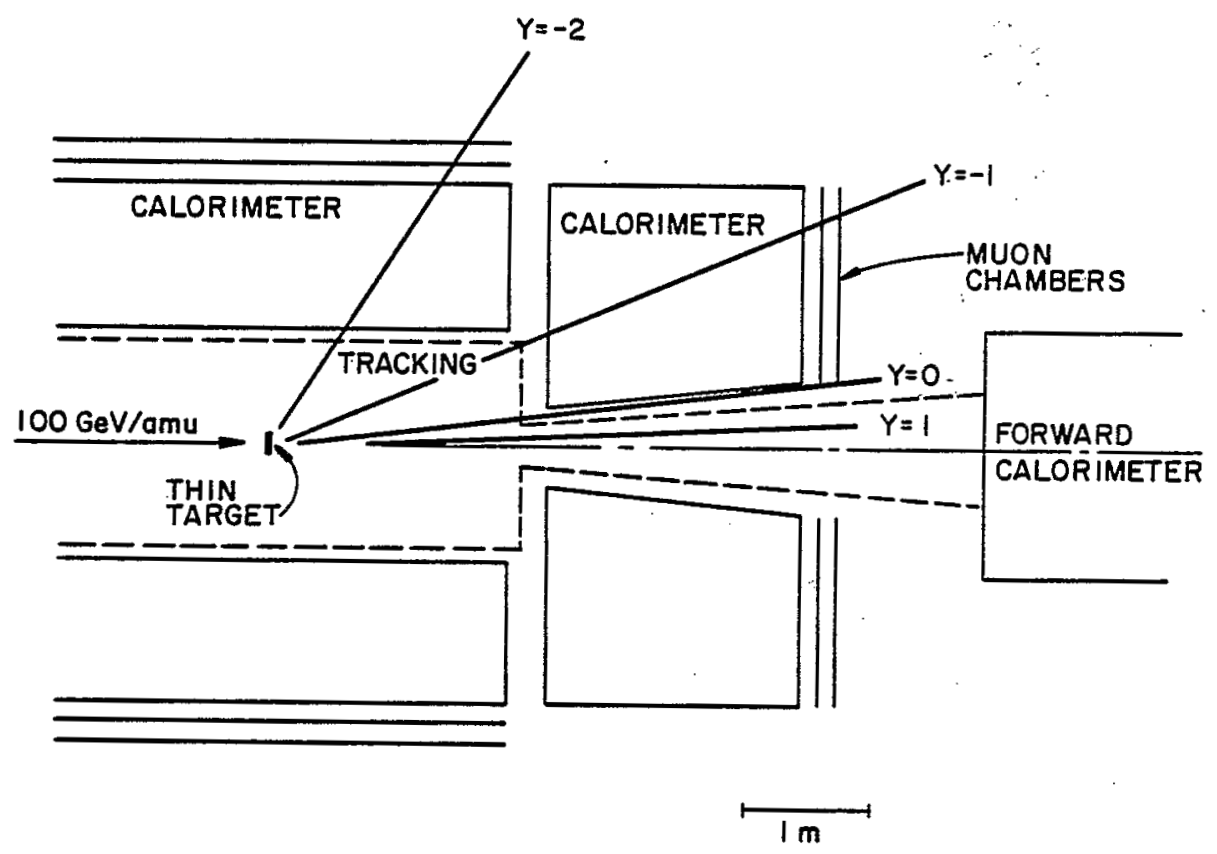

(b) The same detector system, with kinematics of fixed target operation. Here, a single beam at $100 \mathrm{GeV} / \mathrm{amu}$ strikes a thin $\left(\approx 10^{-9} \mathrm{gm} \mathrm{cm}^{-2}\right)$ internal target. 
Fig. $7 b$ shows the same detector configuation being used in a fixed target mode, with only one of the collider beams accelerated and striking a thin target, such as a gas jet. Operation in the fixed target mode--utilizing, if need be, the same detectors as in the collider mode--allows experiments with good luminosity in the lower energy range where the Collider Iuminosities would be unacceptably small. This mode of fixed target operation would not, of course, require the expense of extracted beams, and provides in a natural way (as does collider operation) the extremely thin targets which are crucial for nuclear beams experiments and difficult to realize in conventional fixed target operation with external beams.

A clear difference between high energy experiments with elementary particle beams and those with ultra-relativistic nuclear beams is that in the latter case most of the essential measurements involve soft particles, with transverse momenta and pair masses characteristic of the kinetic energies in a thermalized plasma. These range from near-zero (e.g. coherent photons of a few MeV produced in conjunction with large-scale charge fluctuations) through direct radiation from the plasma in the range $0.5-3 \mathrm{GeV}$. This is in contrast with the elementary particle case where the focus is largely on very rare processes produced in the tails of momentum distributions ( $P_{\mathrm{T}}>10 \mathrm{GeV}$ ) due to hard scattering events, and thus backgrounds from soft particles characterizing the average properties of events are easily eliminated. For nuclear beam experiments, the signals of interest must generally be extracted from the high multiplicity component of soft particles. This also implies that reasonable samples of useful events can be accumulated without the requirement of extraordinarily high interaction rates. For the most massive ions $(A \geq 200)$ the nuclear interaction cross section is 10 barns. 'This means that a machine with luminosity $I=10^{23} \mathrm{~cm}^{-2} \mathrm{sec}^{-1}$ would provide an interaction rate of 1 event/sec. This is already a vast improvement over the present rate of accumulation for high energy cosmic ray events ( $\approx 12$ events/year for the JACEE experiment), 13 but would not allow much flexibility of trigger selection. At $L=10^{24} \mathrm{~cm}^{-2} \mathrm{sec}^{-1}$ we have available one head-on collision per second. With $I \geq 10^{25} \mathrm{~cm}^{-2} \mathrm{sec}^{-1}$ quite sensitive experiments become possible. An upper limit of usable luminosity (again, for the heaviest ions) is probably in the range $L=10^{29} \mathrm{~cm}^{-2} \mathrm{sec}^{-1}$, where the detectors become exposed to a flux of particles $>10^{7} \mathrm{sec}^{-1} \mathrm{ster}^{-1}$. The design luminosity for the proposed 
RHIC facility is $\geq 10^{26} \mathrm{~cm}^{-2} \mathrm{sec}^{-1}$ for $\mathrm{Au}$ beams at $100 \mathrm{GeV}$, as indicated in Fig. 6 .

At the very highest energies, and at luminosities $?$

$10^{26} \mathrm{~cm}^{-2} \mathrm{sec}^{-1}$, a new and potentially very powerful probe of nuclear matter becomes accessible in the form of large transverse momentum jets. These may allow us to study the propagation of elementary quarks and gluons through the high temperature plasma in which chiral symmetry is restored, as well as through ordinary nuclear matter. To do these experiments, we must detect jets among the high multiplicity background of soft particles. This background corresponds to $320 \mathrm{GeV}$ of transverse energy per unit rapidity at $y=0$. The amount of this energy in a cone the size of a jet determines the minimum $P_{T}$ of an observable jet, which is estimated to be $40 \mathrm{GeV} / \mathrm{c}$ for collisions of $A=200$ beams at $100+100 \mathrm{GeV} / \mathrm{amu}$. The estimated rate for observing jets in heavy ion collisions increases very rapidly with increasing beam energy, as illustrated in Fig. 8.

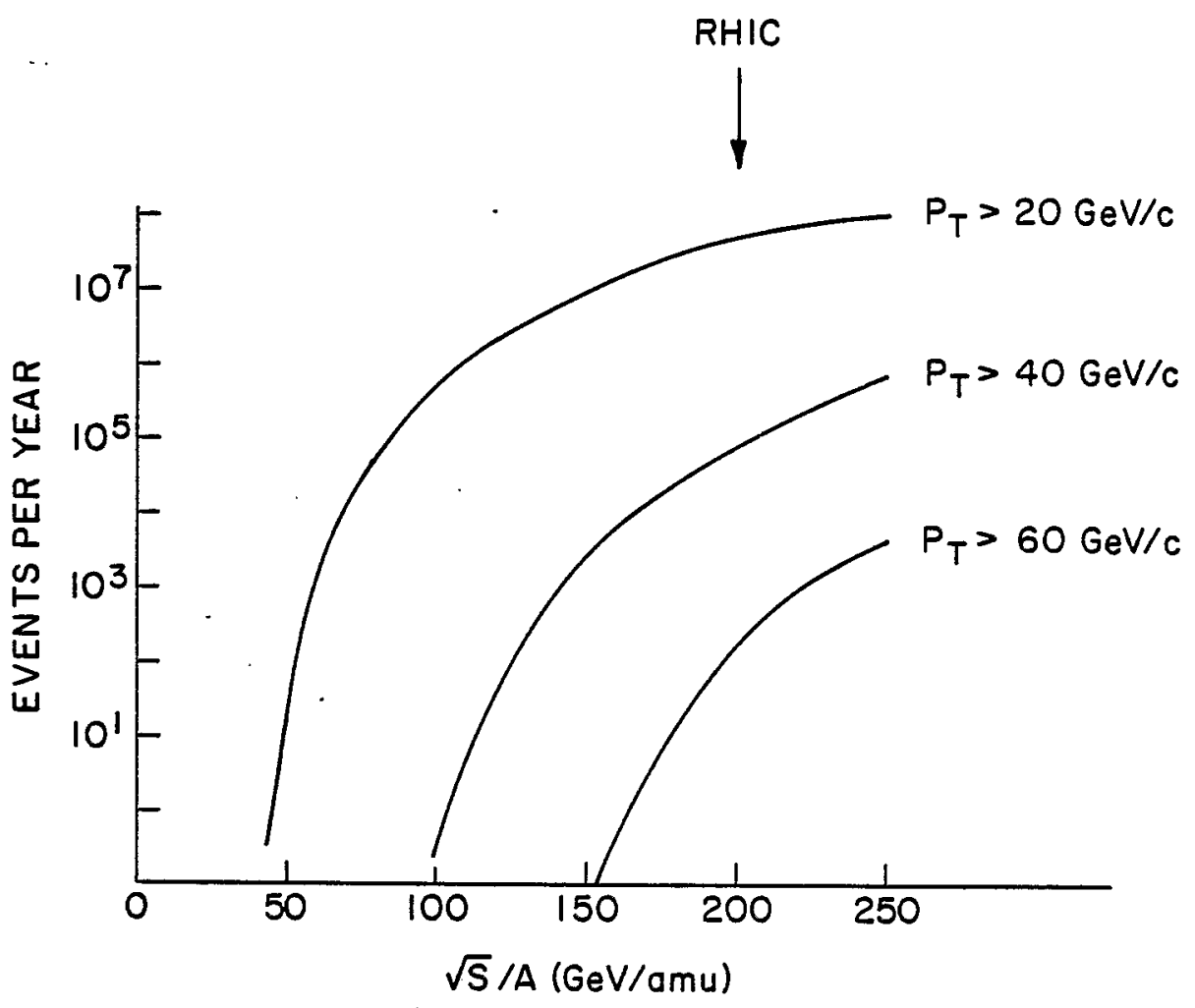

Figure 8

The estimated rates for observing jets at large transverse momentum, as a function of the collision energy. The calculation (Ref. 10) is for gold ions at a Iuminosity $\mathrm{L}=10^{2} \mathrm{~cm}^{-2} \mathrm{sec}^{-1}$. The curves for three different threshold values of $\mathrm{P}_{\mathrm{T}}$ are shown. 